\section{(1) \\ CrossMark}

\title{
Infertility in an adult cohort with primary ciliary dyskinesia: phenotype-gene association
}

\author{
To the Editor:
}

Primary ciliary dyskinesia (PCD) is a rare autosomal recessive disorder (prevalence 1:10000 to 1:40000 births) characterised by impaired mucociliary clearance because of abnormal motile ciliary function $[1,2]$. Five main ultrastructural PCD phenotypes have been described. Most result from a lack of dynein arms (DAs): no outer and inner DAs (2DAs), outer DAs alone (ODA) or inner DAs with microtubular disorganisation (IDA/MTD); or defects yielding an abnormal central complex (CC). Some patients with genetically confirmed PCD have apparently normal ciliary structure on electron microscopy (nEM). More than 30 genes encoding proteins involved in the structure or assembly of the axoneme, the ciliary internal cytoskeleton, are implicated in PCD [3]; their analysis enables identification of bi-allelic disease-causing mutations in $50-75 \%$ of patients. Approximately half of PCD cases are associated with situs inversus, thereby defining Kartagener's syndrome. Moreover, because motile cilia and sperm flagella share common axonemal structures, most PCD-affected males are thought to be infertile [4]. According to the literature, male infertility is caused by severe or total asthenozoospermia and is currently treated by recourse to in vitro fertilisation or intracytoplasmic sperm injection [5, 6]. However, spontaneous fatherhood of PCD patients has been reported.

The prevalence of infertility among PCD females is unclear [4]. Some authors suggested that more frequent ectopic pregnancies could reflect altered motility of Fallopian tube cilia [7]. However, RAIDT et al. [8] described nine female PCD patients with severely dysfunctional respiratory cilia who conceived spontaneously and delivered babies.

Hypothesising a potential association between infertility risk in males and females with PCD and genotype because translated protein expressions vary in different human tissues, we evaluated the fertility of a large adult cohort with well-phenotyped and well-genotyped PCD patients recruited from the Center of Rare Pulmonary Diseases (RESPIRARE) in France and the Dept of Pneumology at the University Center of Leuven, Belgium.

We retrospectively recorded the fertility status of adults with definite PCD diagnosis between January 2014 and January 2016. Every patient's ultrastructural phenotype was well documented and the genotype was established based on the identification of two nonambiguous mutations in known PCD gene(s). The ciliary phenotype was determined in respiratory airway samples obtained from the inferior nasal turbinate or a bronchus, as previously described [9]. All the coding regions and intronic boundaries of all known PCD genes related to each ultrastructural phenotype in genomic DNA were studied by Sanger sequencing (primers available upon request) or next-generation sequencing for those $>30$ exons. All PCR and sequencing primers were designed to avoid polymorphisms with an allele frequency $>0.1 \%$ in control populations exceeding 1000 individuals from the 1000 Genomes database. Our institution's ethics review board approved this study (CCTIRS no. 08.015bis) and all patients gave informed consent.

Fertility data were systematically evaluated by the treating physician during a routine outpatient visit or from patients' files. Spontaneous conception defined normal fertility. Failure to conceive spontaneously for $\geqslant 1$ year and/or when pregnancy was obtained after assisted reproductive technologies (ART) defined infertility [10]. For the statistical analysis, a comparison between patients with and without infertility was performed using the Chi-squared or the Fisher test (as appropriate) for the study of independence among categorical variables.

@ERSpublications

Infertility, observed in $75 \%$ of male and $61 \%$ of female PCD patients, is dependent on ultrastructural and gene defects http://ow.ly/P4K030fPnPp

Cite this article as: Vanaken GJ, Bassinet L, Boon M, et al. Infertility in an adult cohort with primary ciliary dyskinesia: phenotype-gene association. Eur Respir J 2017; 50: 1700314 [https://doi.org/10.1183/ 13993003.00314-2017]. 
Among the 167 adult PCD patients (74 women, 93 men) recruited, only 85 (50.9\%) had fertility data available at the time of evaluation (table 1; supplementary data is available on request from the authors). Among the remaining 83, 61 had not yet tried to conceive and no information on their sexual activity was collected. Patients with available data were older than those without fertility information (median (range) ages 40.5 (33.3-47.0) versus 30.0 (23.5-35.0) years, respectively).

Among the 36 women included, 14 (38.9\%) had spontaneously conceived at a median age of 36 (28-39) years and delivered 31 children. The miscarriage rate was $8 \%$. No ectopic pregnancy was reported. Among $22(61.1 \%)$ women considered infertile (median age 43 (36-46) years), six became pregnant after ART, yielding six live births.

Among the 49 males, 12 (24.5\%) had spontaneously fathered a child (median age 34.5 (33-40.2) years) and 37 (75.5\%) were infertile (median age (range) 42.5 (35-52) years). Among the latter, 15 benefitted from ART, which yielded 22 children.

Cilia motility, evaluated for 64 patients, revealed no difference between fertile and infertile patients (Chi-squared $=1.34, \mathrm{p}=0.25$ ).

When classified according to EM, the percentage of infertile patients differed significantly (Fisher's exact test, $\mathrm{p}=0.005)$. Patients with IDA/MTD and 2DA defects were more likely to be infertile. On the other hand, patients with other EM defects, e.g. ODA, CC or nEM, had a higher chance of being spontaneously fertile. Analysis of the different genes involved, in groups of three or more patients, showed that patients with mutations in CCDC39 or CCDC40 (associated with IDA/MTD defects), those with mutations in DNAAF1 (LRRC50) or LRRC6 (associated with 2DA defects) were more likely to be infertile; those with RSPH4A mutations were more likely to be fertile. However, we did not identify any statistical association between the genotype leading to a potential protein synthesis and fertility (Chi-squared $=0.73, \mathrm{p}=0.39$ ).

To our knowledge, fertility of PCD female patients has never been thoroughly reported. Our study is the first evaluating fertility as a function of known gene defects in a large adult PCD cohort. Despite data being based on patients' responses to questionnaires and retrospective chart analyses, and the sperm status

\begin{tabular}{|c|c|c|c|c|c|c|}
\hline \multirow{2}{*}{$\begin{array}{l}\text { Ciliary defect } \\
\text { (EM) }\end{array}$} & \multirow[t]{2}{*}{ PCD gene } & \multirow{2}{*}{$\begin{array}{l}\text { Patients } \\
\text { (n=85) }\end{array}$} & \multicolumn{2}{|c|}{ Men (n=49) } & \multicolumn{2}{|c|}{ Women $(n=36)$} \\
\hline & & & $\begin{array}{l}\text { Fertile } \\
\text { (n=12) }\end{array}$ & $\begin{array}{c}\text { Infertile } \\
\text { (n=37) }\end{array}$ & $\begin{array}{l}\text { Fertile } \\
\text { (n=14) }\end{array}$ & $\begin{array}{c}\text { Infertile } \\
\text { (n=22) }\end{array}$ \\
\hline \multirow[t]{6}{*}{ CC } & DNAJB13 & 1 & 0 & 1 & 0 & 0 \\
\hline & HYDIN & 6 & 1 & 1 & 0 & 4 \\
\hline & RSPH1 & 4 & 0 & 1 & 1 & 2 \\
\hline & RSPH3 & 2 & 0 & 1 & 0 & 1 \\
\hline & RSPH4A & 3 & 3 & 0 & 0 & 0 \\
\hline & RSPH9 & 2 & 0 & 0 & 1 & 1 \\
\hline \multirow[t]{3}{*}{ IDA/MTD } & CCDC39 & 8 & 0 & 7 & 0 & 1 \\
\hline & CCDC40 & 10 & 1 & 8 & 0 & 1 \\
\hline & GAS8 & 1 & 0 & 1 & 0 & 0 \\
\hline \multirow[t]{4}{*}{ ODA } & DNAH5 & 7 & 3 & 1 & 2 & 1 \\
\hline & DNAI1 & 5 & 0 & 3 & 2 & 0 \\
\hline & NME8 & 1 & 0 & 0 & 0 & 1 \\
\hline & (TXNDC3) & & & & & \\
\hline \multirow[t]{7}{*}{ 2DAs } & DYX1C1 & 2 & 0 & 2 & 0 & 0 \\
\hline & DNAAF1 & 4 & 0 & 1 & 0 & 3 \\
\hline & (LRRC50) & & & & & \\
\hline & LRRC6 & 6 & 0 & 4 & 0 & 2 \\
\hline & $R P G R$ & 1 & 1 & 0 & 0 & 0 \\
\hline & SPAG1 & 1 & 0 & 1 & 0 & 0 \\
\hline & ZMYND10 & 1 & 0 & 1 & 0 & 0 \\
\hline $\mathrm{nEM}$ & DNAH11 & 17 & 3 & 4 & 7 & 3 \\
\hline \multirow[t]{2}{*}{ Others } & CCNO & 2 & 0 & 0 & 1 & 1 \\
\hline & MCIDAS & 1 & 0 & 0 & 0 & 1 \\
\hline
\end{tabular}

PCD: primary ciliary dyskinesia; CC: abnormal central complex; IDA/MTD: inner dynein arms with microtubular disorganisation; ODA: no outer dynein arms; 2DAs: no inner and outer dynein arms; nEM: no EM-detected defect. 
of a female patient's partner not always known, the following conclusions can be drawn. The primary female infertility rate $(63 \%)$ was high, compared with that of the general population $(<30 \%$ of women $44-$ 45 years old) [11]. Although the patients' cilia motility was altered, no ectopic pregnancy occurred in our cohort and the miscarriage rate was $8 \%$, which is even lower than the $15 \%$ observed in the general population. Our male patients were not strictly infertile, since $24 \%$ of them spontaneously fathered a child.

Our findings illustrate a potential link between fertility status, ultrastructure and the involved gene(s). Therefore, this information makes it important to include fertility in patient counselling. According to the literature, among the 90 families harbouring CCDC39 or CCDC40 mutations, involved in IDA/MTD, fertility data were available for 17 adults (three women and 14 men, including some of our patients); all of them were infertile $[12,13]$. We confirmed those observations, as 17 of our 18 patients with mutations involving those genes were infertile. Notably, the only fertile patient in the IDA/MTD subgroup carries a homozygous hypomorphic CCDC40 mutation, c.552G>A; p.(Leu184=), which is predicted to partially modify without completely disrupting the intron-3 donor splice site. Hence, the amount of protein produced by that patient is probably not null. Similarly, the four patients with DNAAF1 (LRRC50) and the six with LRRC6 mutations (2DA group) were 100\% infertile [14-16]. We confirmed that finding herein: 10 patients with DNAAF1 and LRRC6 mutations were also infertile. Taken together, these results suggest that PCD patients with mutations in one of these four genes (i.e. CCDC39, CCDC40, DNAAF1 or LRRC6) are very likely to be infertile. These clinical findings correspond well with tissue protein expression data provided by the Human Protein Atlas. All four genes have been shown to have high selective expression levels not only in epithelial cells of the airways, but also in Fallopian tubes and testicular cells. All our findings might be important for ART issues, since sperm quality in known to decay over time, and sperm cryopreservation could be proposed to young PCD patients.

Limitations of the study are the retrospective collection of information. In addition, the lack of fertility data for 22 patients illustrates that, despite these patients being followed in referral centres, physicians do not always collect fertility data and, moreover, highlights the difficult in assessing fertility, especially in women. Indeed, although men can request sperm analysis, women have no way to test their potentially PCD-related fertility before trying to conceive.

In summary, in contrast to what is often suggested in the literature, PCD does not implicitly/always cause infertility; it only implies an increased risk of fertility problems in both sexes. Furthermore, ART techniques are helpful for most male and female patients. PCD-related fertility, especially for women, might go undetected for a long time. Therefore, all PCD specialists should be aware of these issues. Fertility counselling should be included in standard PCD patient care, in close collaboration with fertility specialists. For women, it is difficult because no simple technique is available to evaluate the motility of Fallopian tube cilia. Finally, a larger prospective study on ultrastructural phenotype and genotype, including the gene(s) involved and the existence of at least one hypomorphic mutation, and fertility-related parameters (e.g. smoking and body mass index), would contribute to better evaluation for the fertility prognoses of PCD patients.

Gert Jan Vanaken ${ }^{1,12}$, Laurence Bassinet ${ }^{2,12}$, Mieke Boon ${ }^{3}$, Rahma Mani ${ }^{1}$, Isabelle Honorée ${ }^{4}$, Jean-Francois Papon ${ }^{5,6}$, Harry Cuppens ${ }^{7}$, Martine Jaspers ${ }^{7}$, Natalie Lorent ${ }^{8}$, André Coste ${ }^{6,9}$, Estelle Escudier ${ }^{1,6,10}$, Serge Amselem ${ }^{1,10}$, Bernard Maitre $^{2,6}$, Marie Legendre ${ }^{1,10,13}$ and Sophie Christin-Maitre ${ }^{9,11,13}$

${ }^{1}$ Service de Génétique et Embryologie Médicales, Hôpital Armand-Trousseau, Assistance Publique-Hôpitaux de Paris, Paris, France. ${ }^{2}$ Centre Hospitalier Intercommunal de Créteil, Service de Pneumologie et de Pathologie Professionnelle, DHU A-TVB, Université Paris Est-Créteil, Créteil, France. ${ }^{3}$ Dept of Paediatrics, Pediatric of Pneumology, University Hospital Leuven, Leuven, Belgium. ${ }^{4}$ Assistance Publique-Hôpitaux de Paris, Hôpital Cochin, Service de Pneumologie, Université Paris Descartes, Sorbonne Paris Cité, Paris, France. ${ }^{5}$ Assistance Publique-Hôpitaux de Paris, Hôpital de Bicêtre, Service d'ORL, Faculté de Médecine du Kremlin-Bicêtre, Université Paris Sud, Orsay, France. ${ }^{6}$ Institut National de la Santé et de la Recherche Médicale (INSERM), UMR_S955, Equipe 13, Univ Paris Est-Créteil, Créteil, France. ${ }^{7}$ Dept of Otorhinolaryngology, Head and Neck Surgery, University Hospital Leuven, Leuven, Belgium. ${ }^{8}$ Dept of Respiratory Disease, University Hospital Leuven, Leuven, Belgium. ${ }^{9}$ Service d’ORL, Hôpital Intercommunal de Créteil, DHU A-TVB, Université Paris Est-Créteil, Créteil, France. ${ }^{10}$ INSERM, Unité Mixte de Recherche S933, Université Pierre et Marie Curie, Paris, France. ${ }^{11}$ Service d'Endocrinologie et Maladies de la Reproduction, Hôpital St-Antoine, Assistance Publique-Hôpitaux de Paris, Paris, France. ${ }^{12}$ These authors contributed equally to this work. ${ }^{13} \mathrm{M}$. Legendre and S. Christin-Maitre jointly supervised this work.

Correspondence: Bernard Maitre, Service de Pneumologie et de Pathologie Professionnelle, Centre Hospitalier Intercommunal de Créteil, 40, avenue de Verdun, 94010 Créteil, France. E-mail: bm.maitre@gmail.com

Received: Feb 142017 | Accepted after revision: Aug 252017

This article has been revised according to the correction published in the December 2017 issue of the European Respiratory Journal.

Conflict of interest: None declared. 
Acknowledgements: We thank all the individuals and their families for their cooperation, as well as all the referring physicians. The researchers participate in the network of COST Action BEAT-PCD (Better Evidence to Advance Therapeutic Options for PCD; no. BM 1407). L. Bassinet, I. Honoré, J.-.F Papon, A. Coste, E. Escudier, S. Amselem, B. Maitre, M. Legendre and S. Christin-Maitre are members of the RadiCONetwork (Inserm, France).

\section{References}

1 Lobo J, Zariwala MA, Noone PG. Primary ciliary dyskinesia. Semin Respir Crit Care Med 2015; 36: 169-179.

2 Praveen K, Davis EE, Katsanis N. Unique among ciliopathies: primary ciliary dyskinesia, a motile cilia disorder. F1000Prime Rep 2015; 7: 36.

3 Tilley AE, Walters MS, Shaykhiev R, et al. Cilia dysfunction in lung disease. Annu Rev Physiol 2015; 77: 379-406.

4 Goutaki M, Meier AB, Halbeisen FS, et al. Clinical manifestations in primary ciliary dyskinesia: systematic review and meta-analysis. Eur Respir J 2016; 48: 1081-1095.

5 Rubino P, Viganò P, Luddi A, et al. The ICSI procedure from past to future: a systematic review of the more controversial aspects. Hum Reprod Update 2016; 22: 194-227.

6 Dávila Garza SA, Patrizio P. Reproductive outcomes in patients with male infertility because of Klinefelter's syndrome, Kartagener's syndrome, round-head sperm, dysplasia fibrous sheath, and "stump" tail sperm: an updated literature review. Curr Opin Obstet Gynecol 2013; 25: 229-246.

$7 \quad$ Blyth M, Wellesley D. Ectopic pregnancy in primary ciliary dyskinesia. J Inst Obstet Gynaecol 2008; 28: 358.

8 Raidt J, Werner C, Menchen T, et al. Ciliary function and motor protein composition of human fallopian tubes. Hum Reprod Oxf Engl 2015; 30: 2871-2880.

9 Tamalet A, Clement A, Roudot-Thoraval F, et al. Abnormal central complex is a marker of severity in the presence of partial ciliary defect. Pediatrics 2001; 108: E86.

10 Practice Committee of American Society for Reproductive Medicine. Definitions of infertility and recurrent pregnancy loss. Fertil Steril 2008; 90: S60.

11 Chandra A, Copen CE, Stephen EH. Infertility and impaired fecundity in the United States, 1982-2010: data from the National Survey of Family Growth. Natl Health Stat Rep 2013; 67: 1-18.

12 Antony D, Becker-Heck A, Zariwala MA, et al. Mutations in CCDC39 and CCDC40 are the major cause of primary ciliary dyskinesia with axonemal disorganization and absent inner dynein arms. Hum Mutat 2013; 34: $462-472$.

13 Blanchon S, Legendre M, Copin B, et al. Delineation of CCDC39/CCDC40 mutation spectrum and associated phenotypes in primary ciliary dyskinesia. J Med Genet 2012; 49: 410-416.

14 Duquesnoy P, Escudier E, Vincensini L, et al. Loss-of-function mutations in the human ortholog of Chlamydomonas reinhardtii ODA7 disrupt dynein arm assembly and cause primary ciliary dyskinesia. Am J Hum Genet 2009; 85: 890-896.

15 Loges NT, Olbrich H, Becker-Heck A, et al. Deletions and point mutations of LRRC50 cause primary ciliary dyskinesia due to dynein arm defects. Am J Hum Genet 2009; 85: 883-889.

16 Kott E, Duquesnoy P, Copin B, et al. Loss-of-function mutations in LRRC6, a gene essential for proper axonemal assembly of inner and outer dynein arms, cause primary ciliary dyskinesia. Am J Hum Genet 2012; 91: 958-964. 